\title{
Transcobalamin l: a novel prognostic biomarker of neoadjuvant chemotherapy in locally advanced hypopharyngeal squamous cell cancers
}

This article was published in the following Dove Press journal: OncoTargets and Therapy

\author{
Ying Wang, ${ }^{1,2}$ Changli Yue, ${ }^{3}$ \\ Jugao Fang, ',4,5 Lili Gong, ${ }^{2}$ \\ Meng Lian,' Ru Wang,' Ling \\ Feng,' Hongzhi Ma,' Zhihong \\ $\mathrm{Ma},{ }^{3}$ Honggang $\mathrm{Liu}^{3}$ \\ 'Department of Otorhinolaryngology \\ Head and Neck Surgery, Beijing Tongren \\ Hospital, Capital Medical University, Beijing, \\ People's Republic of China; ${ }^{2}$ Department \\ of Otolaryngology, Liaocheng People's \\ Hospital and Liaocheng Ophthalmological \\ and Otolaryngological Hospital, Liaocheng \\ Clinical School of Taishan Medical \\ University, Liaocheng, Shandong, People's \\ Republic of China; ${ }^{3}$ Department of \\ Pathology, Beijing Key Laboratory of \\ Head and Neck Molecular Diagnostic \\ Pathology, Beijing Tongren Hospital, \\ Capital Medical University, Beijing, People's \\ Republic of China; ${ }^{4} \mathrm{Key}$ Laboratory of \\ Otorhinolaryngology Head and Neck \\ Surgery, Ministry of Education, Beijing \\ Institute of Otorhinolaryngology, Beijing, \\ People's Republic of China; ${ }^{5}$ Beijing Key \\ Laboratory of Head and Neck Molecular \\ Diagnostic Pathology, Beijing, People's \\ Republic of China
}

Correspondence: Jugao Fang Department of Otorhinolaryngology Head and Neck Surgery, Beijing Tongren Hospital, Capital Medical University, I Dongjiaomin Alley, Dongcheng, Beijing 100730, People's Republic of China

$\mathrm{Tel}+861058269911$

$\mathrm{Fax}+861058266699$

Email fangjugaol965।II0@।63.com

Honggang Liu

Department of Pathology, Beijing Key Laboratory of Head and Neck Molecular Diagnostic Pathology, Beijing Tongren Hospital, Capital Medical University, I Dongjiaomin Alley, Dongcheng, Beijing 100730, People's Republic of China

Tel +86 I0 58269911

Fax +86 10 58268685

Email liuhgII25@I63.com
Background: Hypopharyngeal squamous cell carcinoma (HPSCC) is an aggressive head and neck squamous cell carcinoma with poor prognosis. Neoadjuvant chemotherapy (NACT) followed by concurrent chemoradiotherapy could provide better efficacy in HPSCC treatment. Identification of predictive biomarkers is critically needed to improve selection of patients who derive the most benefit from NACT. The aim of this study was to investigate whether transcobalamin I (TCN1) could be a novel predictive biomarker for NACT in HPSCC.

Methods: We collected biopsy specimens from 102 patients with primary locally advanced HPSCC. Messenger RNA (mRNA) and protein expression levels of TCN1 were analyzed using quantitative polymerase chain reaction and immunohistochemistry, respectively. The relationship between TCN1 expression, chemotherapy sensitivity, and clinical outcome was assessed using univariate Kaplan-Meier survival analyses and multivariate analysis with covariate adjustments. Furthermore, we knocked down TCN1 by small interfering RNA (siRNA) in HPSCC cell FaDu, tested the effects of TCN1 knockdown on cisplatin toxicity by MTT assay, and detected cisplatin-induced apoptosis by Western blotting.

Results: TCN1 expression was significantly lower in NACT-sensitive patients than nonsensitive patients at protein level $(p=0.013)$ and mRNA level $(p<0.001)$, indicating that low TCN1 expression predicts better NACT treatment response. Furthermore, TCN1 was an independent prognostic biomarker for both overall survival $(p=0.047)$ and disease-free survival ( $p=0.05$ ) in advanced HPSCC patients. In addition, in vitro experiments showed that genetic silencing of TCN1 using siRNA sensitized FaDu cells to cisplatin treatment with increased cell apoptosis.

Conclusion: Low expression of TCN1 might be a novel prognostic biomarker for predicting NACT sensitivity and clinical outcome in local advanced HPSCC patients.

Keywords: transcobalamin I, hypopharyngeal squamous cell carcinoma, neoadjuvant chemotherapy, prognosis biomarker

\section{Introduction}

Hypopharyngeal squamous cell cancer (HPSCC), a malignancy of hypopharynx, accounts for $\sim 10 \%$ of all head and neck cancer. About $80 \%$ of HPSCC cases are at an advanced stage when diagnosed, that is, the patients are in stage III or IV, for which treatment becomes difficult, and have poor prognosis with a high proportion of distant metastasis $(60 \%)$ compared with other head and neck cancers. ${ }^{1}$ A retrospective analysis showed that the 2-year disease-free survival (DFS) and 2-year overall survival (OS) rates were only $29.3 \%$ and $32.8 \%$, respectively, in patients with stage III/IV hypopharyngeal cancer treated with chemoradiation therapy. ${ }^{2}$ 
In recent years, neoadjuvant chemotherapy (NACT) has been proposed as an alternative proven approach, which significantly reduces the incidence of distant metastasis of locally advanced hypopharyngeal cancer. ${ }^{3}$ A Phase II trial evaluating efficacy after treatment with NACT, followed by concomitant chemoradiotherapy in 59 patients with hypopharynx cancer or base of tongue cancer, reported 3-year OS of $64 \%$ and DFS of $57 \%$ in all patients. ${ }^{4}$ However, resistance to chemotherapeutic agents is a rather common occurrence that is often attributed to poor response and prognosis. Therefore, identifying factors predicting tumor response to NACT and better survival rates would contribute for better personalized treatment.

Transcobalamin 1 (haptocorrin or vitamin B12 [cobala$\mathrm{min}], \mathrm{TCN} 1$ ) is a vitamin B12-binding protein that carries vitamin B12 which passes through the stomach and enzymatically releases it in the gut, where it is subsequently bounded by an intrinsic factor. ${ }^{5-7}$ High TCN1 levels are associated primarily with disorders of granulocyte proliferation, and TCN1 has been found to be overexpressed in several malignancies and correlated to tumor progression and metastasis, such as leukemia, hepatocellular carcinoma, breast cancer, lung cancer, stomach carcinomas, and colon cancer. ${ }^{6-11}$ More recently, a study on bioinformatics by Chu et al has reported that TCN1 could be an important oncogene for the classification of normal and colorectal cancer tissues. ${ }^{12}$ However, little is known about TCN1 expression and clinical relevance in HPSCC.

In this study, the expression of TCN1 in 102 HPSCC patients was first analyzed. Then, the association of TCN1 expression with NACT response and clinical outcome in HPSCC patients were evaluated. At last, the biological effects of TCN1 gene were evaluated using HPSCC cell lines.

\section{Materials and methods}

\section{Patients, samples, and ethics statement}

A total of 102 patients with primary hypopharyngeal squamous cell carcinoma (HPSCC) (aged from 43 to 71 years), who underwent docetaxel, cisplatin, 5-fluorouracilbased induction chemotherapy at the Department of Otorhinolaryngology Head and Neck Surgery of the Beijing Tongren Hospital from 2011 to 2015, were recruited for this study and sorted according to their chemotherapeutic sensitivities. Prior to the NACT, tumor tissues were collected before (biopsy sample) or during surgery. HPSCC was confirmed both pathologically and histologically. Patient characteristics including age, TNM stage, and chemotherapy response were shown in Table 1. The study was approved
Table I Correlation of clinicopathological features and TCNI expression

\begin{tabular}{llll}
\hline Characteristic & $\begin{array}{l}\text { TCN I } \\
\text { negative }\end{array}$ & $\begin{array}{l}\text { TCN I } \\
\text { positive }\end{array}$ & p-value \\
\hline $\begin{array}{l}\text { Age (years) } \\
\quad<57\end{array}$ & $28(27.5 \%)$ & $20(19.6 \%)$ & 0.775 \\
$\quad \geq 57$ & $33(32.4 \%)$ & $21(20.6 \%)$ & \\
T stage & & & 0.583 \\
TI/T2 & $7(6.9 \%)$ & $6(5.9 \%)$ & \\
T3/T4 & $55(53.9 \%)$ & $34(33.3 \%)$ & \\
N stage & & & 0.342 \\
No/NI & $21(20.6 \%)$ & $10(9.8 \%)$ & \\
N2/N3 & $4 I(40.2 \%)$ & $30(29.4 \%)$ & \\
Grade & & & 0.034 \\
GI & $13(I 2.7 \%)$ & $2(2.0 \%)$ & \\
G2 & $21(20.6 \%)$ & $11(10.8 \%)$ & \\
G3 & $28(27.5 \%)$ & $27(26.5 \%)$ & \\
NACT response & & & 0.013 \\
Responder (CR+PR) & $5 I(50 \%)$ & $24(23.5 \%)$ & \\
Nonresponder (SD+PD) & II (I0.8\%) & I6 (I5.7\%) & \\
\hline
\end{tabular}

Abbreviations: $C R$, complete remission; $P R$, partial remission; SD, stable disease; $D P$, disease progression; TCNI, transcobalamin I; NACT, neoadjuvant chemotherapy.

by the Ethical Committee of Capital Medical University, Beijing, China. Patients provided their written informed consent in this study.

\section{Tissue microarray (TMA) construction and immunohistochemistry (IHC)}

TMAs were constructed by using a manual tissue array machine, purchased from Beecher Instruments (Woodland, WI, USA). Two tissue cylinders per tumor with a diameter of $0.6 \mathrm{~mm}$ were present in one TMA. Briefly, a section from the TMA was dewaxed with xylene and gradually hydrated. Antigen retrieval was performed by treatment in a pressure cooker for 6 minutes. Anti-TCN1 monoclonal antibody (1:150 dilution, ab95363; Abcam, Cambridge, MA, USA) was incubated at room temperature for 1 hour. Chemiluminescent signals were detected according to the manufacturer's instruction (LSAB ${ }^{\text {TM }}$ 2-kits; Dako Denmark A/S, Glostrup, Denmark). All slides were interpreted by two pathologists who were blinded to the clinical information at the time of review. IHC staining was scored semi-quantitatively as negative (score 0 ), weak (score 1), moderate (score 2), or strong (score 3). For statistical evaluation, scores 0 and 1 were considered low TCN1 expression, while scores 2 and 3 were considered high TCN1 expression.

\section{Assessment of the response to the chemotherapy regimen}

Head and neck contrast-enhanced 64-slice spiral computed tomography (CT) was performed before and after NACT to 
evaluate the effect of the treatment regimen. Postoperative pathological examinations were performed for the patients who underwent surgery. The response was categorized as complete remission (CR), partial remission (PR), stable disease (SD), and disease progression (DP). Patients with $\mathrm{CR}$ and $\mathrm{PR}$ response were classified as the response group, while patients with SD and DP response were classified as the nonresponse group.

\section{Cell culture and chemicals}

The HPSCC FaDu cells were cultured in RPMI-1640 (Thermo Fisher Scientific, Waltham, MA, USA) containing $10 \%(\mathrm{v} / \mathrm{v})$ heat-inactivated fetal bovine serum (Thermo Fisher Scientific), $100 \mathrm{U} / \mathrm{mL}$ penicillin, and $100 \mu \mathrm{g} / \mathrm{mL}$ streptomycin (Thermo Fisher Scientific). Cells were incubated at $37^{\circ} \mathrm{C}$ in a $5 \% \mathrm{CO}_{2}$ humidified atmosphere. Cisplatin (Sigma-Aldrich Co., St Louis, MO, USA) was dissolved in dimethyl sulfoxide. Anti-TCN1 antibody were purchased from Abcam, while anti-PARP, anti-cleaved-caspase 3, and anti-GAPDH antibodies were purchased from Bioworld Technology (Minneapolis, MN, USA).

\section{RNA interference}

The human TCN1 small interfering RNA (siRNA) duplexes (three unique 27 mers) were purchased from OriGene Technologies (Beijing, China). They targeted three regions of TCN1 messenger RNA (mRNA) (\#1: 5'-ACUCUGUG AGAAUCAAUGAAACATA-3'; \#2: 5'-GAAGGCAGU UUAAAGAACAUCAGTA-3'; and \#3: 5'-GAAUUGCCAA CAAACUCUGAAUACA-3') for interference. A universal scrambled siRNA duplex was used as a negative control. Transfection with TCN1 siRNA duplexes was performed using lipofectamine 2000 (Thermo Fisher Scientific) according to manufacturer's instruction.

\section{Quantitative real-time polymerase chain reaction (PCR) analysis}

As previously described, ${ }^{13}$ total RNA was isolated from 23 patients' frozen tumor specimens, and FaDu cells, using Trizol Reagent (Thermo Fisher Scientific) according to the manufacturer's instruction. One microgram total RNA was reverse transcribed into cDNA using Rever-Tra-Ace$\alpha$-Transcriptase (Toyobo, Tokyo, Japan) and subsequently amplified by PCR using the SYBR ${ }^{\circledR}$ Premix Ex Taq ${ }^{\mathrm{TM}}$ II (Tli RNaseH Plus; Takara, Tokyo, Japan). $\beta$-Actin served as an internal control to normalize the RNA abundance. Quantitative PCR (qPCR) was performed on a LightCycler 480 Real-Time PCR system (Hoffman-La Roche Ltd., Basel,
Switzerland). The comparative $\mathrm{Ct}\left(2^{-\Delta \Delta \mathrm{Ct}}\right)$ method was used to calculate the relative-fold changes of mRNA expression in different groups. The primer sequences are as follows: TCN1: 5'-CCCCTAGTGGGGCTCTTACT-3' (forward) and 5'-CAGAGGTTTTAGGCGGATGTAG-3' (reverse); $\beta$-actin: 5'-AGAGCTACGAGCTGCCTGAC-3' (forward) and 5'-AGCACTGTGTTGGCGTACAG-3' (reverse). All reactions were performed in triplicate, and values are expressed as means $\pm \mathrm{SD}$.

\section{MTT cell proliferation assay and cytotoxicity assay}

As previously reported, ${ }^{14}$ MTT assay was performed according to manufacturer's instructions (Sigma-Aldrich Co.). Briefly, 24 hours after siRNA transfection, cells were plated in 96-well plates, at a density of 2,000 cells per well in $100 \mu \mathrm{L}$ medium. For cell proliferation assay, cells were cultured for 1,3 , and 5 days. For cisplatin toxicity assay, after overnight culture, cells were treated with increasing doses of cisplatin. After 72 hours, $10 \mu \mathrm{L}$ of MTT solution was added to each well and incubated for 3 hours. The absorbance was then measured at a wave length of $570 \mathrm{~nm}$. Cell viability was expressed as a percentage of the control cells, and the IC50 values were calculated from nonlinear regression using the program GraphPad Prism 5.0 software (GraphPad Software, Inc., La Jolla, CA, USA). This experiment was performed three times.

\section{Western blot analysis}

As we did before, ${ }^{13} \mathrm{FaDu}$ cells were collected for protein extraction 48 hours after siRNA transfection or 72 hours after cisplatin treatment. The FaDu cell lysates were lysed with $2 \times$ sodium dodecyl sulfate (SDS) sample buffer $(100 \mathrm{mM}$ Tris- $\mathrm{HCl}[\mathrm{pH}$ 6.8], $10 \mathrm{mM}$ ethylenediaminetetraacetic acid, 4\% SDS, 10\% glycine). After protein quantification with Bicinchoninic Acid kit (Bio-Rad Laboratories Inc., Hercules, CA, USA), $20 \mu \mathrm{g}$ proteins were loaded, separated on a $10 \%$ SDS-PAGE gel, and transferred to a polyvinylidene fluoride membrane. After blocking with 5\% non-fat milk for 1 hour at room temperature, the membranes were incubated with anti-TCN1 $(1: 1,000$, Abcam), anti-PARP $(1: 1,000)$, anti-cleaved-caspase 3 (1:500), and anti-GAPDH (1:300) overnight at $4^{\circ} \mathrm{C}$. The membranes were then washed with TBST three times for 5 minutes each time. Then the membranes were incubated with a horseradish peroxidase-conjugated secondary antibody at room temperature for 1 hour. After washing three times, the signals were detected using ECL kit (Bio-Rad Laboratories Inc.). Each experiment was repeated three times. 


\section{Annexin V/propidium iodide (PI) staining apoptosis assay by flow cytometry}

FaDu cells were plated into 12 -well plates at $1 \times 10^{5} / \mathrm{mL} /$ well and were treated with siRNA transfection. After 24 hours, cells were treated with or without $3 \mu \mathrm{M}$ cisplatin for 72 hours. Cell apoptosis was assessed by double staining with Annexin V-fluorescein isothiocyanate (Nanjing KeyGen Biotech, Nanjing, Jiangsu, China) and PI (50 $\mathrm{g} / \mathrm{mL}$, Sigma-Aldrich Co.). The cells were then immediately analyzed on BD FACSCalibur (BD Biosciences, San Jose, CA, USA). All flow cytometry data were analyzed with FlowJo V7.6 software (FlowJo, LLC, Ashland, OR, USA). All experiments were repeated three times.

\section{Statistical analysis}

The statistical analysis was performed by using the software package SPSS 19.0 (IBM Corporation, Armonk, NY, USA). Chi-square or Fisher's exact test was performed to analyze the statistical significance of the association of TCN1 expression with chemotherapy response, as well as the clinicopathological characters. Univariate Kaplan-Meier analysis and multivariate logistic regression analysis with covariate adjustments were used. The Student's $t$-test was performed to evaluate the differences between TCN1 knockdown and the control cells. A $p$-value of $<0.05$ was considered statistically significant.

\section{Results}

Association of the immunohistochemical expression of TCNI with

\section{clinicopathological features}

Clinicopathological characteristics of the 102 HPSCC patients evaluated for the tumor TMA are presented in Table 1. All recruited patients were treated with NACT regimen, and the response was evaluated before and after NACT treatment by contrast-enhanced 64-slice spiral CT. Seventy-five patients showed good response to NACT including patients who got complete tumor remission or partial tumor remission, and 27 patients were resistant to NACT treatment including patients with SD or progressed disease.

To evaluate the relationship between TCN1 expression levels and clinicopathological characteristics in HPSCC patients treated with NACT, IHC staining was performed on TMA slides constituted with 102 patient samples. Cytoplasmic expression of TCN1 was successfully scored semi-quantitatively in all examined cases. Representative IHC staining is shown in Figure 1A. Of all the patient tissues that were analyzed, low expression of TCN1 was observed in $62(60.8 \%)$ patients and high TCN1 expression in $40(39.2 \%)$ patients. No correlations between TCN1 expression and age, gender, $\mathrm{T}$ staging, and $\mathrm{N}$ staging (Table $1, p>0.05$ ) were found. However, overexpression
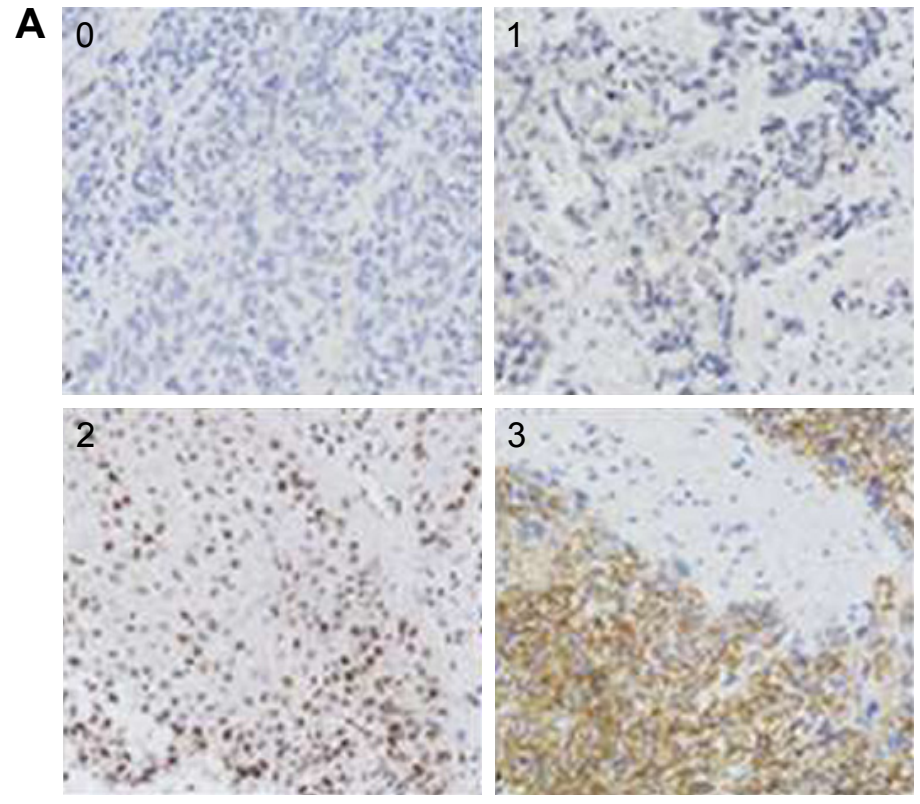

B

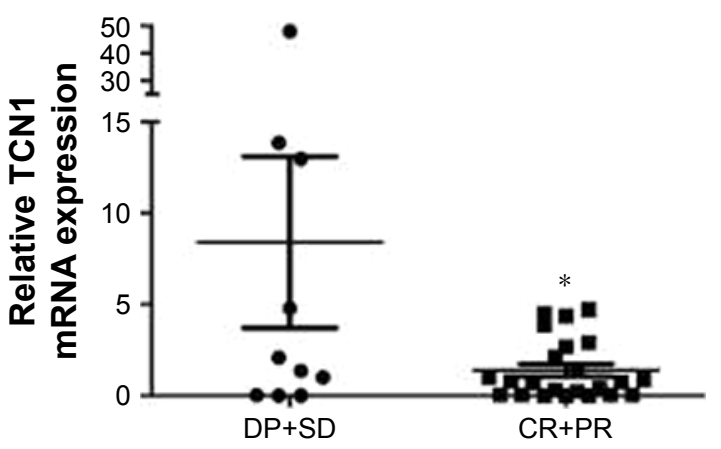

Figure I TCNI protein and mRNA expression in locally advanced hypopharyngeal cancer. (A) Representative of negative (score 0), weak (score I), moderate (score 2), and strong (score 3) TCNI IHC staining on HPSCC tissues. (B) TCNI mRNA expression was analyzed by qPCR in both NACT responders ( $\mathrm{n}=\mathrm{I0}$ ) and nonresponders $(n=23) . * p<0.05$

Abbreviations: mRNA, messenger RNA; IHC, immunohistochemistry; HPSCC, hypopharyngeal squamous cell carcinoma; qPCR, quantitative polymerase chain reaction; TCNI, transcobalamin I; CR, complete remission; PR, partial remission; SD, stable disease; DP, disease progression. 
of TCN1 was correlated with advanced histological grade (Table 1, $p=0.034$ ).

Moreover, TCN1 overexpression was found to be significantly associated with worse response to NACT treatment ( $p=0.013)$. In the group with high TCN1 expression, there were $24(23.5 \%)$ NACT responders (CR or PR) and $16(15.7 \%)$ NACT nonresponders (SD or PD). In the group with low TCN1 expression, by contrast, there were 51 (50\%) NACT responders and 11 (10.8\%) nonresponders. We further isolated mRNA from 33 HPSCC patient tumor specimens, which include 10 NACT responders and 23 NACT nonresponders, and analyzed TCN1 mRNA expression by qPCR. Consistently, qPCR data showed that TCN1 mRNA expression is significantly higher in NACT-resistant group than in sensitive group ( $p=0.029$, Figure 1B).

\section{TCNI acts as a prognostic marker in HPSCC}

In addition, to address whether TCN1 protein expression is an independent prognostic factor for OS and DFS in HPSCC patients, Kaplan-Meier survival analysis was performed. The mean follow-up time of these patients was 23 months (ranged from 2 to 67 months). It was found that there is a trend that high TCN1 expression was correlated with both worse OS (Figure 2A, $p=0.085$ ) and DFS (Figure 2B, $p=0.120$ ). Interestingly, in advanced stage HPSCC patients (T3/T4), high expression of TCN1 was significantly correlated with both OS and DFS ( $p=0.047$ and 0.050 , respectively,
Figure $2 \mathrm{C}$ and $\mathrm{D}$ ). We further performed multivariate analysis in T3/T4 subgroup patients, with common clinical covariates, including gender, $\mathrm{T}$ stage, $\mathrm{N}$ stage, and grade. As shown in Table 2, after adjusting for other covariates, TCN1 expression is still an independent predictor of OS and DFS in local advanced stage HPSCC patients.

\section{Genetic silencing of TCNI suppressed cell growth and increased cisplatin sensitivity FaDu cells}

To investigate the functional role of TCN1 in HPSCC cells, we genetically silenced TCN1 expression in HPSCC FaDu cells, using three siRNA duplexes targeting mRNA encoding human TCN1. A universal scrambled siRNA duplex was used as a control. As presented in Figure 3A and B, transfection with siRNA duplexes led to a marked reduction at both protein and mRNA levels at $48 \mathrm{~h}$ post-transfection, relative to control group. MTT assay demonstrated that downregulation of TCN1 led to a significant reduction in the proliferation of $\mathrm{FaDu}$ cells, relative to control siRNA (Figure $3 \mathrm{C}, p<0.001$ ).

As we described above, the lower level of TCN1 indicated better NACT therapy response. We speculated that suppressing TCN1 expression would enhance the sensitivity of HPSCC cells to cisplatin. To test this hypothesis, an MTT assay was used to examine the impact of cell response to cisplatin by knocking down of TCN1 in FaDu cells. As presented in Figure 4A, TCN1 genetic silencing significantly sensitized FaDu cell to cisplatin treatment, and the cisplatin
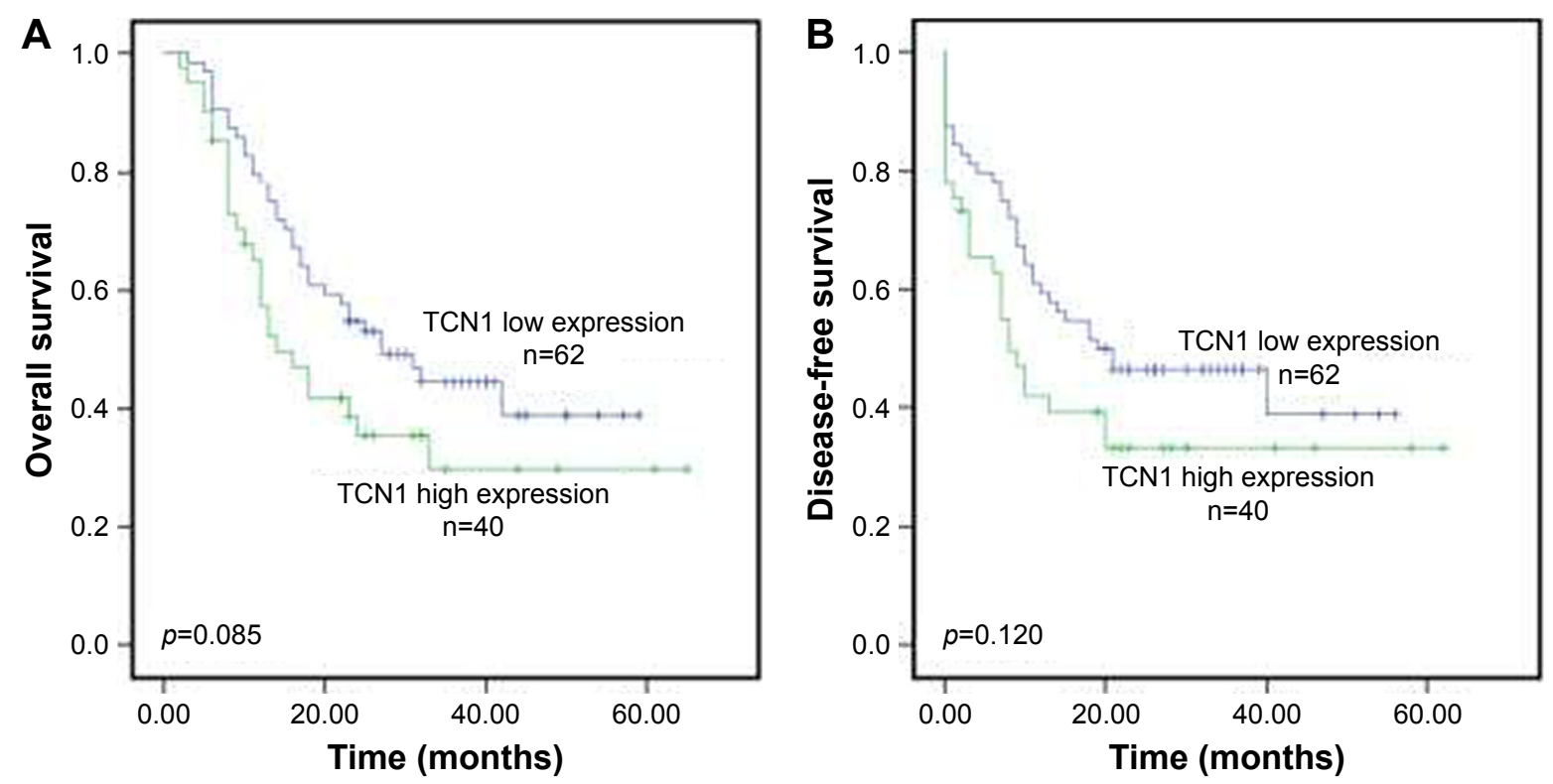

Figure 2 (Continued) 

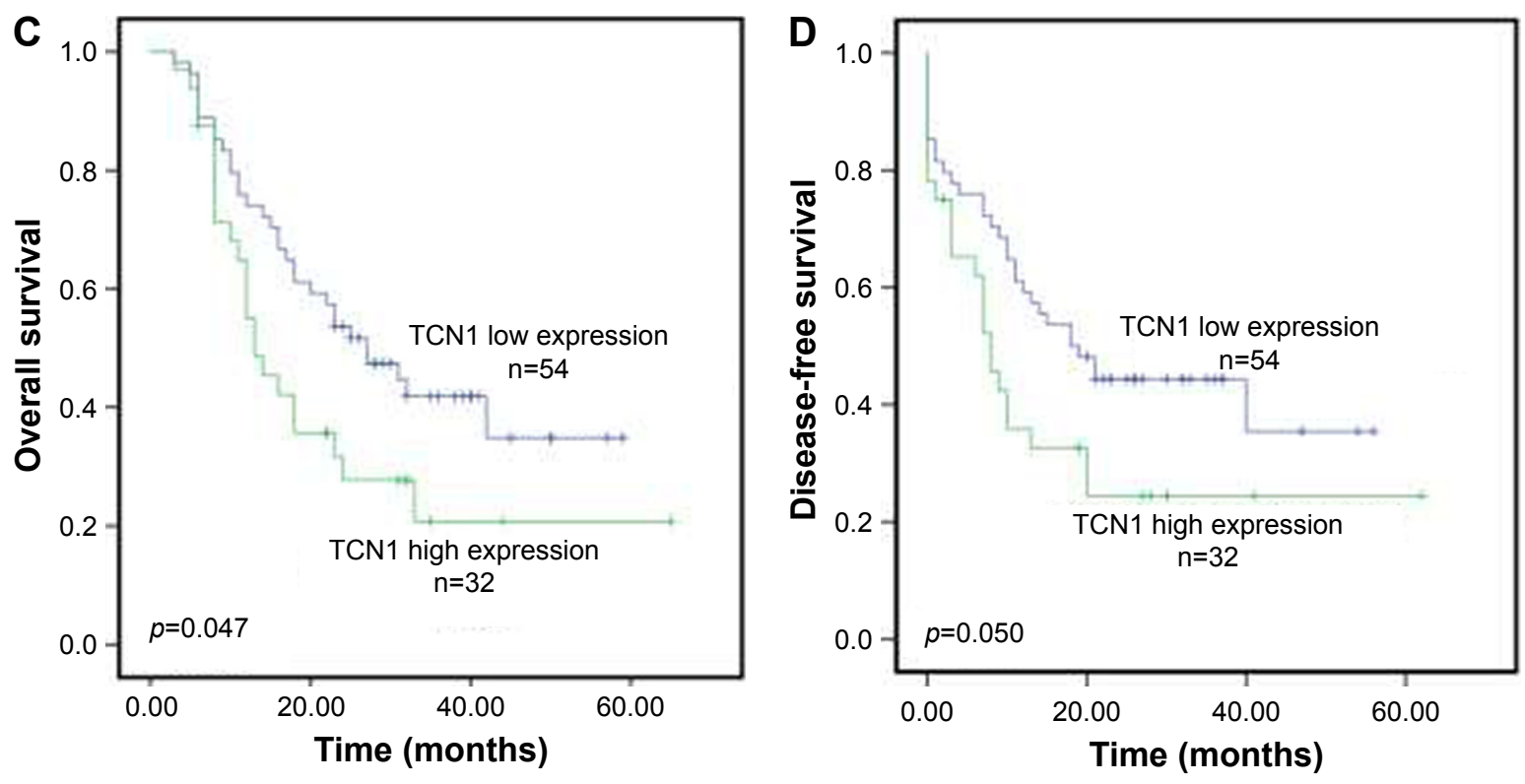

Figure 2 Expression of TCNI predicted clinical outcome in locally advanced hypopharyngeal cancer by Kaplan-Meier curves. (A) Overall survival in whole cohort; (B) disease-free survival in whole cohort; (C) overall survival in patients at stage III/IV; (D) disease-free survival in patients at stage III/IV.

Abbreviation: TCNI, transcobalamin I.

IC50 value significantly decreased to $4.515 \mu \mathrm{M}$ in TCN1 knockdown cells, compared with $6.766 \mu \mathrm{M}$ in control cells. In Figure 4B, Western blot data showed enhanced cleaved caspase- 3 and cleaved PARP after 72 hours cisplatin treatment, indicating increased cell apoptosis in TCN1 knockdown cells compared with control cells. Consistently, Annexin V/PI staining followed by flow cytometry analysis demonstrated that TCN1 knockdown significantly increased the percentage of Annexin V-positive cells when cells were exposed to cisplatin treatment (Figure 4C and D).

\section{Discussion}

During the past decades, there has been a great improvement in the care of patients with HPSCC, and NACT followed by surgery or chemotherapy is the current standard treatment strategy. ${ }^{1-3}$ Prediction of chemotherapy outcomes allows for the personalization of NACT; therefore, a number of clinical

Table 2 Multivariate analyses for OS and DFS of locally advanced hypopharyngeal stage IV cancer patients

\begin{tabular}{|c|c|c|c|c|}
\hline \multirow[t]{2}{*}{ Variables } & \multicolumn{2}{|l|}{ OS } & \multicolumn{2}{|l|}{ DFS } \\
\hline & $\overline{p \text {-value }}$ & $95 \% \mathrm{CI}$ & $\overline{p \text {-value }}$ & $95 \% \mathrm{Cl}$ \\
\hline Gender & 0.960 & $0.346-3.053$ & 0.868 & $0.369-3.267$ \\
\hline T stage & 0.338 & $0.638-3.712$ & 0.365 & $0.623-3.626$ \\
\hline $\mathrm{N}$ stage & 0.191 & $0.772-3.652$ & 0.252 & $0.724-3.424$ \\
\hline Grade & 0.130 & $0.248-1.196$ & 0.171 & $0.266-1.265$ \\
\hline TCNI expression & 0.042 & $1.023-3.475$ & 0.050 & $|.000-3.38|$ \\
\hline
\end{tabular}

Abbreviations: OS, overall survival; DFS, disease-free survival; $\mathrm{Cl}$, confidence interval; TCNI, transcobalamin I. trials have been undertaken to determine the predictive factors for clinical and pathological responses to NACT. Although most patients with advanced head and neck squamous cell carcinoma have poor prognoses, a small subset of patients respond favorably to NACT. The aims of this study were 1) to determine whether TCN1 expression acts as an indicator of response to NACT; 2) to investigate whether TCN1 expression could predict the clinical outcomes of patients with locally advanced hypopharyngeal cancer; and 3) to investigate the biological role of TCN1 in HPSCC cells.

TCN1, a member of the vitamin B12-binding protein family, is a $60-70 \mathrm{kDa}$ molecular weight protein, derived from the granulocyte line. ${ }^{15,16} \mathrm{TCN} 1$ takes part in vitamin B12 homeostasis together with another two vitamin B12 binding proteins, ${ }^{1-3}$ intrinsic factor and TCN2. ${ }^{17}$ High serum B12 levels and TCN1 have been found in patients with various cancers. ${ }^{7,18-21}$ Occasionally, elevated levels of TCN1 were also found in some other myeloproliferative disorders. ${ }^{7,22}$ Mishra et $\mathrm{al}^{23}$ found that the human bone-marrow-derived mesenchymal stem cells grown in tumor-conditioned medium exhibited an upregulation of TCN1 and other carcinoma-associated fibroblast-associated genes. It was also reported that malignant hepatoma and asymptomatic immunodeficiency virus (HIV) seropositive patients presented increased levels of TCN1. ${ }^{22,24}$ Moreover, the protein expression of TCN1 rather than vitamin B12 was found to be better correlated with progression in patients with gastric cancer. ${ }^{9}$ Chu et al recently identified TCN1 as a significantly expressed 
A

B

FaDu
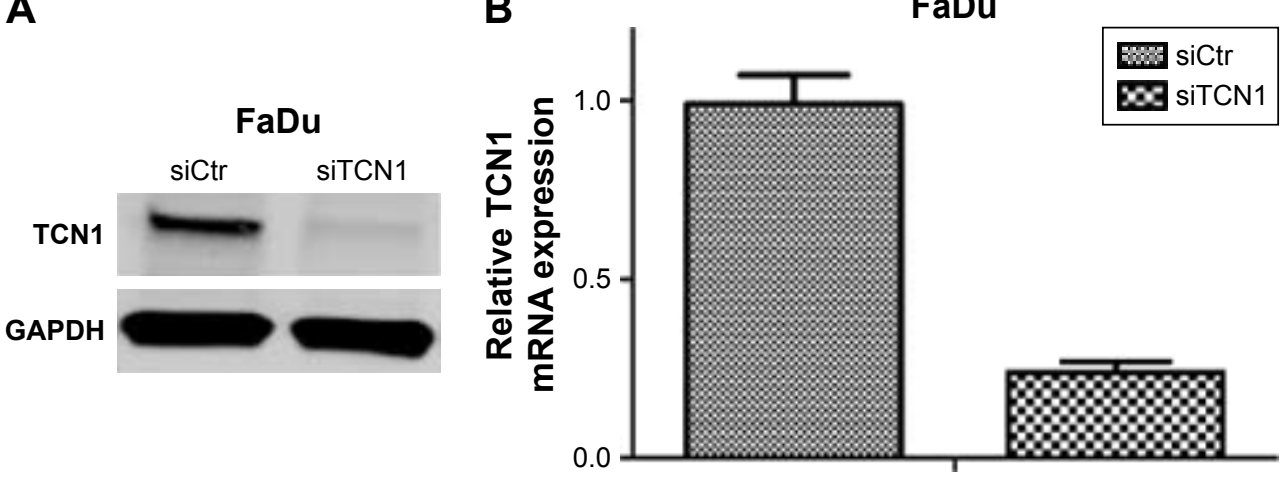

C

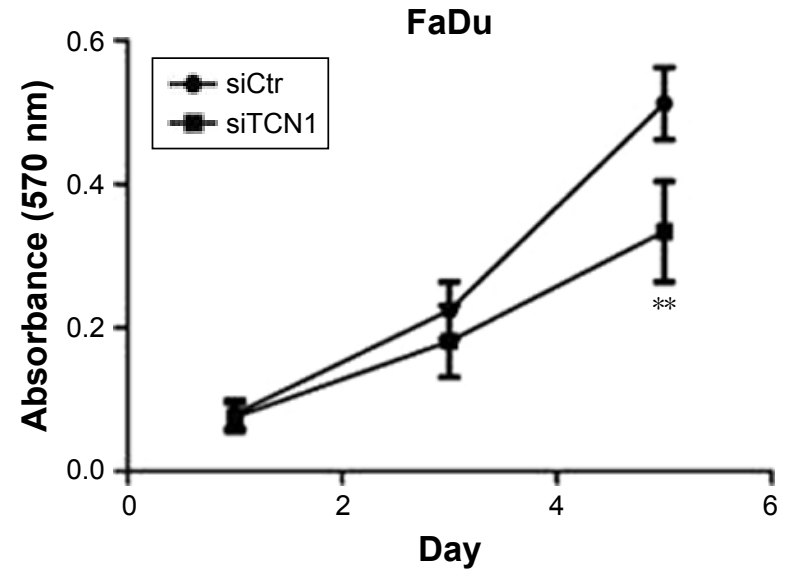

Figure 3 TCNI genetic silencing suppressed HPSCC cell growth. (A) TCNI knockdown in FaDu cells was confirmed at protein level by Western blot and (B) at mRNA level by qPCR. (C) MTT assay showed TCNI knockdown suppressed FaDu cell proliferation. **p $<0.001$

Abbreviations: mRNA, messenger RNA; HPSCC, hypopharyngeal squamous cell carcinoma; qPCR, quantitative polymerase chain reaction; TCNI, transcobalamin I; siCtr, scramble control siRNA; siTCNI, TCNI siRNA.

gene which is associated with advanced colorectal cancer by microarray analysis. ${ }^{12}$ In addition, cytoplasma $\mathrm{TCN} 1$ proved to be a progression marker in fibrolamellar hepatocellular carcinoma. ${ }^{11}$ We present the first report that high expression of TCN1 is positively associated with advanced histological grade and worse prognosis in patients with locally advanced hypopharyngeal cancer in stage IV. These findings indicate that TCN1 might be an important proto-oncogene during the development of hypopharyngeal cancer. Our results are consistent with previous findings regarding the role of TCN1 in various cancers. All the studies performed suggest that TCN1 plays an important biological role in carcinogenesis and tumor progression. However, the underlying mechanisms still need to be further investigated.

NACT is regarded as an efficient treatment for many locally advanced human cancers, including breast and rectal cancer. ${ }^{25,26}$ However, predictive biomarkers for chemosensitivity have rarely been addressed. In this study, mRNA expression of TCN1 that was isolated from patient tumor tissues was significantly higher in patients who were resistant to NACT compared with those who were sensitive to NACT.
Furthermore, these results were confirmed at TCN1 protein expression levels by using IHC. To our knowledge, this is the first report to address that high expression of TCN1 predicted NACT resistance in patients with locally advanced hypopharyngeal cancer. Therefore, TCN1 might serve as an important biomarker for predicting chemosensitivity to NACT.

Consistently, we revealed that the TCN1 knockdown could sensitize HPSCC cell line FaDu to cisplatin treatment, potentially through apoptotic pathway. Besides that, we also found that TCN1 silencing suppressed cell growth. To our knowledge, this study is the first to reveal the biological role of TCN1 in HPSCC.

\section{Conclusion}

To the best of our knowledge, this is the first study to demonstrate that overexpression of TCN1 was closely associated with NACT resistance and an aggressive biological behavior of patients with HPSCC receiving NACT. The strong inverse correlation with survivals in HPSCC suggested that overexpression of TCN1 was an independent negative prognostic biomarker in locally advanced HPSCC. Moreover, 
A

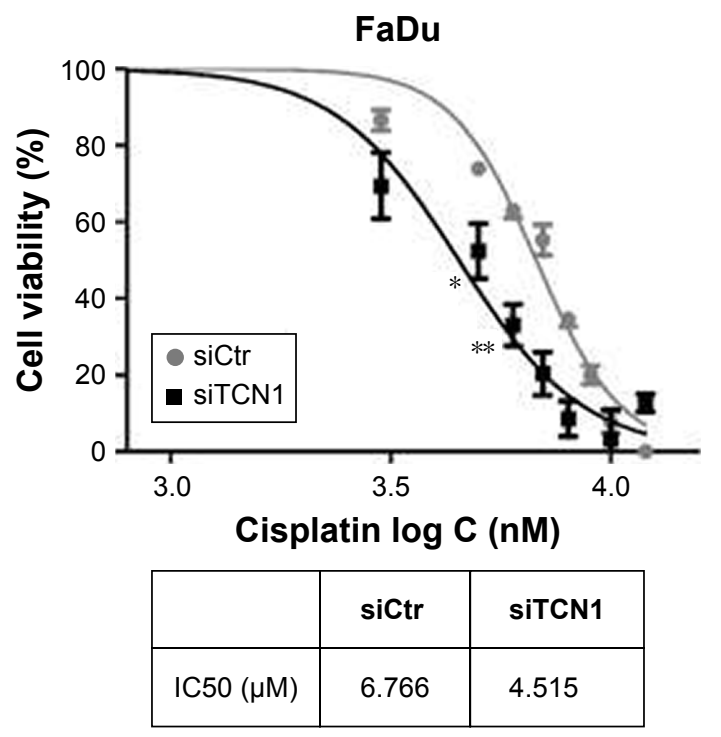

B

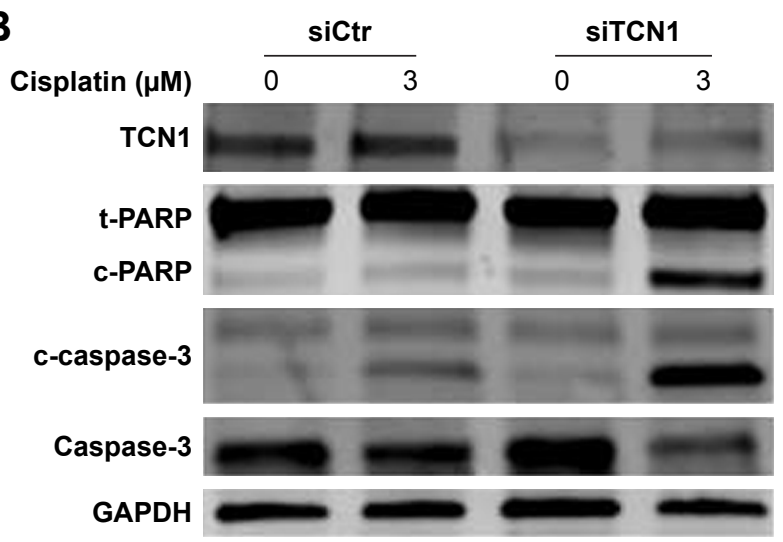

C
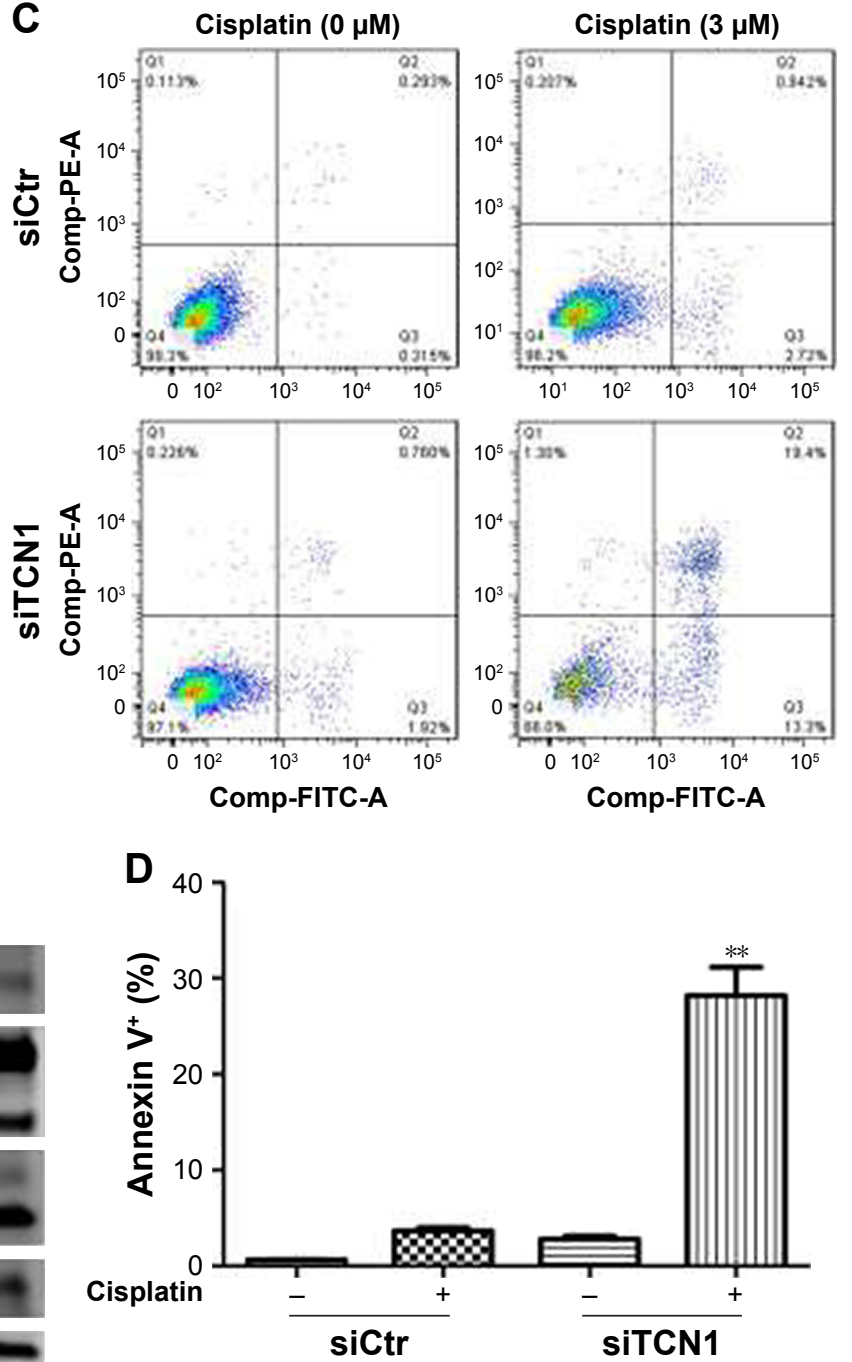

Figure 4 Effects of TCNI downregulation on cisplatin cytotoxicity. (A) Cell viability was reduced after cisplatin treatment in TCNI knocked down FaDu cells. IC50 values are listed below. (B) Western blot analysis of apoptotic pathway-related genes (c-PARP, c-caspase-3) after cells were exposed to cisplatin treatment (3 $\mu$ M). GAPDH was used as a loading control. (C) Flow cytometry analysis of Annexin V and PI staining of cisplatin-induced apoptotic cells in TCNI expressed or knocked down FaDu cells. (D) Percentage of apoptotic cells from flow cytometry experiment. Data represent the mean \pm SEM from three independent experiments. ** $p<0.00 \mathrm{I}$.

Abbreviations: TCNI, transcobalamin I; PI, propidium iodide; SEM, standard error of mean; FITC, fluorescein isothiocyanate; siCtr, scramble control siRNA; siTCNI, TCNI siRNA.

a functional study revealed that TCN1 plays a carcinogenic role in HPSCC. Further understanding of the role of TCN1 in HPSCC and its relationship with NACT efficacy may provide a potential prognostic and therapeutic target, which has clinical significance for the treatment of HPSCC.

\section{Acknowledgments}

This work was supported by Beijing Municipal Science \& Technology Commission Program (no Z141107002514003) and National Natural Science Foundation of China (no 81473499).

\section{Disclosure}

The authors report no conflicts of interest in this work.

\section{References}

1. Hirano S, Tateya I, Kitamura M, et al. Ten years single institutional experience of treatment for advanced hypopharyngeal cancer in Kyoto University. Acta Otolaryngol Suppl. 2010;563:56-61.

2. Krstevska V, Stojkovski I, Lukarski D. Concurrent radiochemotherapy in advanced hypopharyngeal cancer. Radiat Oncol. 2010;5:39.

3. Vishak S, Rangarajan B, Kekatpure VD. Neoadjuvant chemotherapy in oral cancers: selecting the right patients. Indian J Med Paediatr Oncol. 2015;36(3):148-153.

4. Urba SG, Moon J, Giri PG, et al. Organ preservation for advanced resectable cancer of the base of tongue and hypopharynx: a Southwest Oncology Group Trial. J Clin Oncol. 2005;23(1):88-95.

5. Chong LY, Cheok PY, Tan WJ, et al. Keratin 15, transcobalamin I and homeobox gene Hox-B13 expression in breast phyllodes tumors: novel markers in biological classification. Breast Cancer Res Treat. 2012; 132(1):143-151.

6. Waibel R, Treichler H, Schaefer NG, et al. New derivatives of vitamin B12 show preferential targeting of tumors. Cancer Res. 2008;68(8): 2904-2911. 
7. Carmel R. Extreme elevation of serum transcobalamin I in patients with metastatic cancer. N Engl J Med. 1975;292(6):282-284.

8. Gimsing P, Hippe E. Increased concentration of transcobalamin I in a patient with metastatic carcinoma of the breast. Scand J Haematol. 1978;21(3):243-249.

9. Wakatsuki Y, Inada M, Kudo H, et al. Immunological characterization and clinical implication of cobalamin binding protein in human gastric cancer. Cancer Res. 1989;49(11):3122-3128.

10. Ogawa K, Ogawa O, Koshiba M, et al. Immunohistochemical localization of vitamin B12 R-binder in salivary gland tumors. Implications for cell differentiation. Pathol Res Pract. 1990;186(6):751-758.

11. Lildballe DL, Nguyen KQ, Poulsen SS, Nielsen HO, Nexo E. Haptocorrin as marker of disease progression in fibrolamellar hepatocellular carcinoma. Eur J Surg Oncol. 2011;37(1):72-79.

12. Chu CM, Yao CT, Chang YT, et al. Gene expression profiling of colorectal tumors and normal mucosa by microarrays meta-analysis using prediction analysis of microarray, artificial neural network, classification, and regression trees. Dis Markers. 2014;2014:634123.

13. Lian M, Wang H, Fang J, et al. Microarray gene expression analysis of chemosensitivity for docetaxel, cisplatin and 5-fluorouracil (TPF) combined chemotherapeutic regimen in hypopharyngeal squamous cell carcinoma. Chin J Cancer Res. 2017;29(3):204-212.

14. Ma Z, Zhang H, Lian M, et al. SLC7A11, a component of cysteine/ glutamate transporter, is a novel biomarker for the diagnosis and prognosis in laryngeal squamous cell carcinoma. Oncol Rep. 2017;38(5): 3019-3029.

15. Andres E, Serraj K, Zhu J, Vermorken AJ. The pathophysiology of elevated vitamin B12 in clinical practice. QJM. 2013;106(6) $505-515$
16. Gimsing P, Overballe-Petersen C, Hippe E. Cobalamin and cobalaminbinding proteins in plasma related to the clinical condition in chronic myelogenous leukemia. Leukemia. 1995;9(9):1604-1609.

17. Ermens AA, Vlasveld LT, Lindemans J. Significance of elevated cobalamin (vitamin B12) levels in blood. Clin Biochem. 2003;36(8): 585-590.

18. Carmel R, Eisenberg L. Serum vitamin B12 and transcobalamin abnormalities in patients with cancer. Cancer. 1977;40(3):1348-1353.

19. Sheppard K, Bradbury DA, Davies JM, Ryrie DR. Cobalamin and folate binding proteins in human tumour tissue. J Clin Pathol. 1984; 37(12):1336-1338.

20. Meyer LM, Bertcher RW, Cronkite EP, et al. Co60 vitamin B12 binding capacity of serum in persons with hematologic disorders, various medical diseases and neoplasms. Acta Med Scand. 1961;169:557-575.

21. Herbert V. Diagnostic and prognostic values of measurement of serum vitamin B12-binding proteins. Blood. 1968;32(2):305-312.

22. Rule SA, Hooker M, Costello C, Luck W, Hoffbrand AV. Serum vitamin B12 and transcobalamin levels in early HIV disease. Am J Hematol. 1994;47(3):167-171.

23. Mishra PJ, Mishra PJ, Humeniuk R, et al. Carcinoma-associated fibroblast-like differentiation of human mesenchymal stem cells. Cancer Res. 2008;68(11):4331-4339.

24. Fremont $\mathrm{S}$, Champigneulle $\mathrm{B}$, Gerard $\mathrm{P}$, et al. Blood transcobalamin levels in malignant hepatoma. Tumour Biol. 1991;12(6):353-359.

25. King TA, Morrow M. Surgical issues in patients with breast cancer receiving neoadjuvant chemotherapy. Nat Rev Clin Oncol. 2015;12(6): 335-343.

26. Smith JJ, Garcia-Aguilar J. Advances and challenges in treatment of locally advanced rectal cancer. J Clin Oncol. 2015;33(16):1797-1808.
OncoTargets and Therapy

\section{Publish your work in this journal}

OncoTargets and Therapy is an international, peer-reviewed, open access journal focusing on the pathological basis of all cancers, potential targets for therapy and treatment protocols employed to improve the management of cancer patients. The journal also focuses on the impact of management programs and new therapeutic agents and protocols on

\section{Dovepress}

patient perspectives such as quality of life, adherence and satisfaction. The manuscript management system is completely online and includes a very quick and fair peer-review system, which is all easy to use. Visit http://www.dovepress.com/testimonials.php to read real quotes from published authors. 